Revue d'histoire de l'Amérique française

REVUE D.HISTOIRE DE L'AMÉRIQUE FRANÇAISE

\title{
Structure et effectifs des métiers du fer à Montréal avant 1765
}

\section{Dominique Bouchard}

Volume 49, numéro 1, été 1995

URI : https://id.erudit.org/iderudit/305400ar

DOI : https://doi.org/10.7202/305400ar

Aller au sommaire du numéro

Éditeur(s)

Institut d'histoire de l'Amérique française

ISSN

0035-2357 (imprimé)

1492-1383 (numérique)

Découvrir la revue

Citer cette note

Bouchard, D. (1995). Structure et effectifs des métiers du fer à Montréal avant 1765. Revue d'histoire de l'Amérique française, 49(1), 73-85.

https://doi.org/10.7202/305400ar

\section{Résumé de l'article}

L'article qui suit mesure la fiabilité des mentions professionnelles apparaissant dans les actes de baptême, de mariage et de sépulture de la région de Montréal sous le Régime français à partir de données extraites des actes notariés de la colonie. Il estime les effectifs des métiers du fer et l'importance numérique relative de sept d'entre eux (forgeron, taillandier, serrurier, armurier, arquebusier, chaudronnier et ferblantier) et tire certaines conclusions sur l'évolution des appellations relatives aux métiers du fer dans la colonie française d'Amérique du Nord.
Tous droits réservés Institut d'histoire de l'Amérique française, 1995
Ce document est protégé par la loi sur le droit d'auteur. L'utilisation des services d'Érudit (y compris la reproduction) est assujettie à sa politique d'utilisation que vous pouvez consulter en ligne.

https://apropos.erudit.org/fr/usagers/politique-dutilisation/ 


\section{NOTE DE RECHERCHE STRUCTURE ET EFFECTIFS DES MÉTIERS DU FER À MONTRÉAL AVANT 1765' \\ DOMINIQUE BOUCHARD}

\section{RÉSUMÉ}

L'article qui suit mesure la fiabilité des mentions professionnelles apparaissant dans les actes de baptême, de mariage et de sépulture de la région de Montréal sous le Régime français à partir de données extraites des actes notariés de la colonie. Il estime les effectifs des métiers du fer et l'importance numérique relative de sept d'entre eux (forgeron, taillandier, serrurier, armurier, arquebusier, chaudronnier et ferblantier) et tire certaines conclusions sur l'évolution des appellations relatives aux métiers du fer dans la colonie française d'Amérique du Nord.

\section{ABSTRACT}

This paper controls the validity of professional titles appearing in the baptism, marriage and burial acts from the Montreal district under the French Regime using data taken from notarial deeds. It also provides an estimate of the number of metalsmiths active in the area and the numerical importance of seven types of metalsmithing. Finally, observations are made concerning the adaptation of French professional titles to the colonial context.

Pour l'essentiel, l'histoire quantitative des métiers en NouvelleFrance reste à écrire. Ceci entre autres parce que le repérage de ceux qui les exerçaient est des plus difficiles. Moins nombreux que les habitants, moins portés que les marchands à laisser des traces écrites de leur vie professionnelle et privée, les artisans n'ont donné lieu jusqu'à présent qu'à des portraits impressionnistes ${ }^{2}$ certes fort

1. Cette recherche a été réalisée dans le cadre d'un projet dirigé par messieurs Christian Dessureault et John Dickinson, de l'Université de Montréal, et financée par le Conseil national de la recherche.

2. Mentionnons pour mémoire la thèse de doctorat de Peter Moogk, The Craftsmen of New France (Université de Toronto, 1973); l'ouvrage de Louise Dechêne, Habitants et marchands de Montréal au XVIT siècle (Montréal, Boréal Express, 1988); l'étude ethnohistorique de Jean-Claude Dupont, L'artisan forgeron (Québec, Presses de l'Université LavahÉditeur officiel du Québec, 1979); la recherche de Jean-Pierre Hardy et de David-Thierry Ruddel, Les apprentis artisans à Québec, 1660-1815 (Montréal, Presses de l'Université du 
intéressants, mais incapables de nous fournir ne serait-ce qu'un ordre de grandeur de leurs effectifs.

Le Programme de recherche en démographie historique (PRDH) du Département de démographie de l'Université de Montréal exploite un registre informatique de la population du Québec ancien qui comprend notamment toutes les mentions professionnelles apparaissant dans les actes d'état-civil de la colonie. Ces indications pourraient nous aider à mieux cerner l'importance relative et la répartition des métiers en Nouvelle-France si on savait à quel point elles sont fiables. Mais telles qu'elles se présentent, elles sont trop irrégulières et variables pour qu'on puisse par ce seul moyen déterminer à coup sûr qu'un artisan exerçait le métier que le registre du PRDH lui prête.

La base de données Parchemin de la Société Archiv-Histo nous fournit toutefois une source indépendante pour valider les données du PRDH. Elle rassemble en effet des résumés de tous les actes notariés passés sous le Régime français. Il est possible d'en extraire des sousensembles selon diverses clés de tri, dont le métier. Un chercheur est ainsi en mesure de dresser une liste de tous les colons ayant passé devant notaire au moins un acte dans lequel ils s'identifiaient comme artisan. En corrélant cette liste avec les fiches du registre du PRDH, il devient possible d'évaluer la fiabilité des mentions professionnelles des actes d'état-civil. Du moins en théorie. Pour tenter de nous en assurer, nous avons appliqué la démarche au groupe de métiers que nous connaissions le mieux ${ }^{3}$, celui des artisans du fer.

\section{INSTRUMENTS DE TRAVAIL}

Lancé en 1980, le projet Parchemin a pour but de poursuivre le travail des archivistes Pierre-Georges et Antoine Roy. Son initiatrice, la Société de recherche historique Archiv-Histo, s'est associée avec la Chambre des notaires du Québec et les Archives nationales du Québec pour réaliser un inventaire systématique du patrimoine notarial québécois, qui puisse être facilement consulté tant par les chercheurs professionnels que par les généalogistes amateurs. Parchemin est une base de données conviviale où l'utilisateur retrouve, sous forme de courtes rubriques, les éléments essentiels de chacun des actes notariés passés avant la fin de 1765 dans la partie de la Nouvelle-France cor-

Québec, 1977); et la synthèse réalisée sous la direction d'Yves Landry, Pour le Christ et le Roi. La vie au temps des premiers Montréalais (Montréal, Éditions Libre Expression/Art Global, 1992).

3. Pour en avoir fait le sujet de notre mémoire de maitrise, puis celui d'un article pour la Revue d'histoire de l'Amérique française («La culture matérielle des Canadiens au XVIII siècle: analyse du niveau de vie des artisans du fer», RHAF, 47,4 (printemps 1994): 479-498). 
respondant au territoire québécois: nom, prénom et surnom des parties contractantes, selon la graphie originale; âges, métiers et lieux de résidence s'ils figuraient dans l'original; sans oublier les références propres à l'acte lui-même comme la date, le nom du notaire, la nature du contrat, etc. L'orthographe des mentions professionnelles ayant été uniformisée, l'utilisateur qui demande un tri par métier est certain d'obtenir la liste complète des actes dans lesquels une personne a déclaré la profession sélectionnée. Les règles de dépouillement des actes et de rédaction des articles de la base sont exposées dans Parchemin s'explique ${ }^{4}$.

Le registre de population du Québec ancien est l'œuvre d'une équipe multidisciplinaire qui s'est créée en 1968 au Département de démographie de l'Université de Montréal. Le Programme de recherche en démographie historique a reconstitué la population européenne de la vallée du Saint-Laurent des débuts de la colonie française jusqu'à 1800 à partir des registres paroissiaux (actes de baptême, de mariage et de sépulture) et de documents comme les recensements de la période française et les listes d'embarquement de colons. En raison du caractère semi-fermé de cette population relativement petite et du bon état de conservation des archives paroissiales, ce registre démographique est remarquablement complet. N'y échappe qu'une toute petite fraction de la population: les gens qui ne sont pas nés dans la colonie, ne s'y sont pas mariés, n'y ont pas été recensés et n'y sont pas morts. Ceux-là n'ont pas de fiche à leur nom, mais peuvent quand même apparaître comme témoins, sans numéro d'identification propre, dans des actes concernant des individus dûment identifiés. L'information de la base se présente sous forme de fiches de divers types, dont une fiche de famille où figurent les noms des parents, conjoints et enfants du sujet, en plus de ses dates de naissance, de mariage et de décès; et une fiche individuelle qui fournit la liste des actes auxquels la personne a participé comme sujet, parent ou témoin, ainsi que la ou les professions qu'elle a pu s'attribuer à ces occasions et la paroisse où l'événement a eu lieu. L'utilisateur a aussi accès aux transcriptions des actes ${ }^{5}$.

4. Hélène Lafortune, Robert Normand et Serge Goudreau, Parchemin s'explique... Guide de dépouillement des actes notariés du Québec ancien (Montréal, Ministère des Affaires culturelles, Archives nationales du QuébecChambre des notaires du Québec, 1989).

5. Les principes qui ont guidé l'équipe du PRDH ont été exposés par Jacques Légaré dans «Le programme de recherche en démographie historique de l'Université de Montréal: fondements, méthodes, moyens et résultats», Études canadiennes/Canadian Studies, 10 (juin 1981): 149-182. Une mise à jour de ce texte a été publiée en anglais sous le titre «A Population Register for Canada Under the French Regime: Context, Scope, Content and Application», Canadian Studies in Population, 15,1 (1988): 1-16. 


\section{MÉTHODOLOGIE}

La première étape de notre projet a consisté à extraire de la base Parchemin tous les résumés des actes passés par des notaires du gouvernement de Montréal où était mentionné l'un des métiers que nous voulions étudier: forgeron, taillandier, serrurier, armurier, arquebusier, chaudronnier, ferblantier, maréchal, cloutier, fondeur (ces trois derniers par acquit de conscience, car ils n'étaient pas véritablement exercés en Nouvelle-France). Nous avons tiré de chaque acte le nom de famille, le surnom (le cas échéant) et le ou les prénoms du ou des artisans du fer qui y étaient identifiés comme tels ${ }^{6}$, leur lieu de résidence s'ils l'avaient déclaré, la référence notariale (nom du notaire et date de l'acte), l'objet général du contrat (obligation, apprentissage, concession, etc.) et un certain nombre de détails susceptibles de faciliter le repérage de nos sujets dans le registre du PRDH (âge, noms des parents, nom de l'épouse). Nous avons uniformisé l'orthographe des noms de famille en vue du tri automatique, mais avons noté la graphie du notaire parmi ces observations générales.

Nous avons dressé une liste distincte pour chaque métier dans l'ordre suivant: forgeron, taillandier, serrurier, armurier, arquebusier, ferblantier, chaudronnier et divers. Cet ordre a son importance. Il arrive assez fréquemment, en effet, qu'un artisan se donne plusieurs titres professionnels dans le même acte (par exemple, forgeron et taillandier, armurier et arquebusier, chaudronnier et ferblantier). Pour éviter de répéter ces entrées d'une liste à l'autre, nous avons éliminé de celle des taillandiers toutes les références à un forgeron-taillandier (puisque l'acte avait été pris en compte dans le registre des forgerons), de celle des serruriers toutes les mentions à un forgeron-serrurier ou à un taillandier-serrurier, et ainsi de suite; les mentions professionnelles supplémentaires ont été consignées dans la liste du premier métier déclaré (selon l'ordre ci-dessus), avec l'orthographe originale du nom et les autres détails d'identification. Le nombre total d'entrées dans une liste ne correspond donc au nombre réel d'actes extraits de la base Parchemin que dans un cas, celui des forgerons.

Cette recension faite, nous avons trié les entrées selon deux clés alphabétiques - le nom de famille et le prénom - et une clé chronologique - l'année de l'acte (afin de faciliter la différenciation des homonymes). Ensuite, nous avons établi une fiche pour chacun des artisans que nous pouvions identifier, y transcrivant son nom, avec

6. Il nous est arrivé de repérer dans un acte un artisan dont nous connaissions le métier par un acte antérieur, mais qui ne l'avait pas déclaré au notaire à cette occasion. Nous n'avons pas inclus ces cas dans la liste. 
toutes ses variantes orthographiques, son surnom s'il en avait un, les prénoms qu'il avait employés, le nombre d'actes que nous pouvions lui attribuer, les dates du premier et du dernier de ces actes, le ou les lieux de résidence mentionnés selon les années, les métiers déclarés et les autres indices disponibles.

Enfin, nous avons tenté de retracer dans le registre du PRDH les artisans de nos fiches sans faire appel aux mentions professionnelles (puisque c'était ce qu'il fallait valider).

\section{STRUCTURE ET EFFECTIFS DES MÉTIERS DU FER D'APRÉS LES ACTES NOTARIÉS}

Les 1937 actes de la liste des forgerons nous ont livré 407 noms pour le gouvernement de Montréal (419 en comptant une douzaine d'artisans résidant dans les régions de Québec ou de Trois-Rivières, selon leur déclaration au notaire), ce qui représente une moyenne de cinq actes par nom. Pour les autres catégories professionnelles, on obtient les chiffres suivants: taillandiers, 806 actes et 153 noms; serruriers, 181 actes et 38 noms; armuriers, 267 actes et 62 noms; arquebusiers, 227 actes et 33 noms; ferblantiers, 82 actes et 15 noms; chaudronniers, 63 actes et 19 noms; divers (cloutier, maréchal, fondeur), 17 actes et 9 noms. Les écarts sont évidemment magnifiés par l'élimination des actes en double, mais il demeure que le groupe des forgerons se détache très nettement des autres et que celui des serruriers paraît un peu réduit compte tenu de l'importance que devrait avoir ce métier par rapport à la ferblanterie et à la chaudronnerie, par exemple.

Une proportion considérable de ces noms se retrouve dans deux, trois, voire quatre fichiers. Recoupements faits, les 736 noms des huit listes se réduisent à 541 individus. Parmi eux, pas moins de 241 (45\%) figurent dans un seul acte: il s'agit dans 129 cas d'un brevet d'apprentissage. Les 112 autres correspondent à des contrats de mariage, de concession, de vente ou d'achat, à des engagements pour la traite, à des obligations, etc. Les sujets de ces actes uniques ne pouvaient être intégrés à notre échantillon de validation puisque le risque était grand qu'ils n'aient jamais pratiqué ou n'aient exercé que très brièvement dans la région montréalaise avant de partir ou de changer de profession. Ce qui nous laissait 300 sujets potentiels.

Plus on a d'actes sur un artisan, plus on dispose d'information spatiale, chronologique ou sociale sur lui, et meilleures sont les chances de l'identifier sans risque d'erreur. Parmi les 300 artisans restants, 56 figuraient dans deux actes, 28 dans trois et 216 dans au moins quatre actes. Un échantillon de plus de 200 sujets nous paraissait 
amplement suffisant pour nos fins, et l'existence d'au moins quatre actes nous semblait offrir de bonnes garanties pour le repérage (et pour l'exercice continu du métier dans la région de Montréal). Nous avons donc limité le travail de couplage aux 216 artisans qui nous avaient laissé quatre actes ou plus.

De ces artisans confirmés, 176 figurent dans la liste des forgerons, 103 dans celle des taillandiers, 25 dans celle des serruriers, 34 dans celle des armuriers, 19 dans celle des arquebusiers, 7 dans celle des ferblantiers, 9 dans celle des chaudronniers. Les «divers» ajoutent deux noms, pour un total de 375 qui est bien supérieur à 216 parce que beaucoup de sujets s'attribuent deux ou trois métiers différents ${ }^{7}$.

Lorsqu'on procède au recoupement de ces sept listes, on constate que les artisans du fer montréalais se divisent non pas en sept métiers, mais en trois catégories inégales. La première, qui rassemble $85 \%$ des effectifs (183 sujets sur 216), est composée de touche-à-tout que le langage tend à réunir sous le vocable générique de forgeron ${ }^{8}$. La deuxième regroupe les $10 \%$ de notre échantillon (22 sujets) qui se spécialisaient dans l'entretien des armes. La troisième est des plus restreintes, à peine 11 personnes $(5 \%)$ pour toute la période française, et concerne des métiers très spécifiques, qui devaient générer une demande assez faible mais stable, la chaudronnerie et la ferblanterie. Essayer d'établir des distinctions fines entre forgerons, taillandiers et serruriers serait peine perdue ${ }^{9}$. En ce qui concerne les spécialités, l'armurerie et l'arquebuserie sont confondues dans les faits comme dans la langue; par contre, les chaudronniers semblent conserver une personnalité relativement distincte des ferblantiers (on relève dans ces deux métiers d'authentiques dynasties, les Lauzon pour la

7. Seulement sept taillandiers sur 103 ne se disent jamais forgeron, et aucun des 25 serruriers ne se réclame de ce seul métier. Chez les 34 armuriers, en revanche, on trouve six hommes qui se cantonnent à l'armurerie et 12 qui n'ajoutent à cette pratique que celle de l'arquebuserie. Quant aux 19 arquebusiers, ils s'identifient si étroitement aux armuriers que 16 d'entre eux se disent aussi armurier. Un seul ne se trouve dans aucune liste sauf la sienne. Enfin, les ferblantiers et les chaudronniers semblent former une petite confrérie assez fermée. Des sept ferblantiers, deux ne s'imputent aucun autre métier, et cinq se disent chaudronnier, tandis que cinq chaudronniers sur neuf portent exclusivement ce titre, trois n'y associent que celui de ferblantier et seulement un se déclare forgeron (entre autres choses!).

8. Ce schéma a, comme toujours, ses exceptions. Ambroise Casale dit Lalime, par exemple, apparaît dans une cinquantaine d'actes. Il s'y déclare parfois forgeron parfois taillandier, ce qui le fait entrer dans la catégorie des généralistes. En fait, dans une écrasante majorité des cas, il opte pour le titre de serrurier, signe que c'est à ce métier-là qu'il estimait appartenir. Quant à savoir s'il le pratiquait vraiment dans les années 1730-1740 à Sault-auRécollet, c'est une autre histoire.

9. Le recoupement est tel que neuf forgerons sur dix emploient le titre de taillandier, et qu'à peine sept pour cent des taillandiers évitent systématiquement celui de forgeron; quant à la serrurerie, elle est quasiment annexée par les forgerons-taillandiers. 
Tableau 1

Répartition de l'échantillon $(n=216)$ selon le nombre d'appellations

\begin{tabular}{lrrrrr}
\hline Nombre d'appellations & 1 & 2 & 3 & 4 & 5 \\
Nombre de sujets & 54 & 122 & 32 & 7 & 1 \\
Pourcentage de l'effectif & 25,0 & 56,5 & 14,8 & 3,2 & 0,5 \\
\hline
\end{tabular}

Tableau 2

Répartition des 54 appellations uniques par métier

\begin{tabular}{lrrrrrrrr}
\hline Titre de métier & $\mathrm{fg}$ & $\mathrm{tl}$ & $\mathrm{sr}$ & ar & aq & fr & ch & $\mathrm{dv}$ \\
Nombre de sujets & 36 & 3 & 0 & 6 & 1 & 2 & 5 & 1 \\
Pourcentage du total & 66,7 & 5,6 & 0,0 & 11,1 & 1,9 & 3,7 & 9,3 & 1,9 \\
\hline
\end{tabular}

Tableau 3

Répartition des 162 appellations multiples selon la présence de forgeron

Deux métiers Trois métiers Quatre métiers Cinq métiers

\begin{tabular}{lrrrrrrrr}
\hline Avec forgeron & 103 & $84,4 \%$ & 30 & $93,8 \%$ & 6 & $85,7 \%$ & 1 & $100 \%$ \\
Sans forgeron & 19 & $15,7 \%$ & 2 & $6,2 \%$ & 1 & $14,3 \%$ & 0 & $0 \%$ \\
\hline
\end{tabular}

Tableau 4A

Répartition des 103 appellations doubles comportant forgeron

\begin{tabular}{lrrrrrrr}
\hline Doubles: fg+ & $\mathrm{tl}$ & $\mathrm{sr}$ & $\mathrm{ar}$ & $\mathrm{aq}$ & $\mathrm{fr}$ & $\mathrm{ch}$ & $\mathrm{dv}$ \\
Nombre de sujets & 89 & 6 & 6 & 1 & 0 & 0 & 1 \\
Pourcentage du total & 86,4 & 5,8 & 5,8 & 1,0 & 0,0 & 0,0 & 1,0 \\
\hline
\end{tabular}

Tableau 4B

Répartition des 30 appellations triples comportant forgeron

\begin{tabular}{lccc}
\hline Métiers & & Sujets & $\%$ \\
\hline fg+tl+ & sr & 18 & 60,0 \\
fg+tl+ & ar & 9 & 30,0 \\
fg+tl+ & dv & 1 & 3,3 \\
fg+ar+ & sr & 2 & 6,6 \\
\hline
\end{tabular}


Tableau 4C

Répartition des 6 appellations quadruples comportant forgeron

\begin{tabular}{lccc}
\hline Métiers & & Sujets & $\%$ \\
\hline $\mathrm{fg}+\mathrm{tl}+\mathrm{sr}+$ & ar & 2 & 33,3 \\
$\mathrm{fg}+\mathrm{t}+\mathrm{sr}+$ & $\mathrm{ch}$ & 2 & 33,3 \\
$\mathrm{fg}+\mathrm{tl}+\mathrm{sr}+$ & $\mathrm{dv}$ & 1 & 16,7 \\
$\mathrm{fg}+\mathrm{tl}+$ & $\mathrm{ch}+\mathrm{fr}$ & 1 & 16,7 \\
\hline
\end{tabular}

Tableau 5

Répartition des 22 appellations multiples ne comportant pas forgeron

\begin{tabular}{llcc}
\hline Doubles & Sujets & $\%$ \\
\hline & & 12 & 63,2 \\
& ar+aq & 4 & 21,0 \\
& fr+ch & 3 & 15,8
\end{tabular}

Triples

$\begin{array}{lll}(\mathrm{ar}+\mathrm{aq})+\mathrm{tl} & 1 & 50,0 \\ (\mathrm{ar}+\mathrm{aq})+\mathrm{sr} & 1 & 50,0\end{array}$

Quadruples

$(\operatorname{ar}+\mathrm{aq})+\mathrm{tl}+\mathrm{sr}$

1

100

Dans les tableaux 1 à 5 , nous employons les codes suivants pour désigner les différents métiers: fg pour forgeron; tl pour taillandier; sr pour serrurier; ar pour armurier; aq pour arquebusier; fr pour ferblantier; ch pour chaudronnier; dv pour divers (maréchal, fondeur, cloutier).

chaudronnerie, et les Beaumont Pistolet pour la ferblanterie) quoique dans la pratique, les empiétements dussent être courants.

\section{COMPARAISON AVEC LES DONNÉES DES ACTES D'ÉTAT-CIVIL}

Après avoir repéré dans le registre informatique du PRDH les fiches individuelles des 216 artisans titulaires d'au moins quatre actes dans la base Parchemin, nous avons relevé les mentions professionnelles lorsqu'elles y figuraient.

Des 183 membres du groupe des forgerons-taillandiers-serruriers, 98 portent le code 20 qui, dans le système du PRDH, identifie les 
forgerons ${ }^{10}, 24$ un autre code 2 (qui signifie qu'ils travaillaient le métal), 12 un code numérique différent de 2 (le plus souvent un 7 de commerçant, un 9 de militaire ou un 1 de cultivateur); 48 fiches ne fournissent aucun code de métier. Quant au dernier sujet, il ne figure pas dans la base. En somme, le registre du PRDH fournit une indication générique utile (à savoir un code signalant un métier du fer) deux fois sur trois pour le groupe des généralistes ${ }^{11}$.

Dans le groupe des 22 armuriers-arquebusiers, 18 portent le code 21 qui désigne ces deux professions (le PRDH ne fait pas la distinction); trois n'ont pas le code 2 des métiers du fer. Un deuxième isolé complète la distribution. La corrélation est meilleure que celle qu'on observe dans le premier groupe, $82 \%$, et elle vaut même au niveau spécifique du métier.

Elle semble du même ordre pour les ferblantiers: six fiches individuelles sur sept (86\%) ont le code $2 \mathrm{~m}$, et ce code n'est accompagné d'aucun autre, ce qui confirme le caractère exclusif du métier. (Le septième sujet n'a jamais rien confessé au curé de sa paroisse.) Mais $2 \mathrm{~m}$ désigne aussi bien les ferblantiers que les chaudronniers dans la base démographique. Or, la situation est plus complexe pour les chaudronniers. Seulement deux, Gilles Lauzon et son fils Séraphin, portent le code $2 \mathrm{~m}$; les deux autres n'ont rien à signaler. Il faut dire que l'un d'eux est mort très jeune ( 22 ans) si bien que sa fiche individuelle est avare de détails. De plus, l'échantillon est tellement réduit qu'il suffirait d'ajouter une fiche pour modifier substantiellement le taux de corrélation. Les corrélations spécifique et générique sont à nouveau égales puisque $2 \mathrm{~m}$ est le seul code 2 relevé.

En gros, le Registre de population du Québec ancien rate un artisan du fer sur trois chez les généralistes, mais seulement un spécialiste sur cinq, et identifie correctement les armuriers, ferblantiers et chaudronniers qu'il recense. Autrement dit, si une fiche individuelle du registre fait mention d'un 2 , on peut légitimement postuler que le sujet a exercé un métier du fer. Si ce code est un 21 ou un $2 \mathrm{~m}$, il est très probable que le métier en question ait été l'armurerie ou la ferblanterie-chaudronnerie, surtout si le code figure à répétition ou sans autre code 2; par contre, il est inutile de chercher à trancher,

10. Et quelques autres comme les maréchaux-ferrants et les poêliers. Ce sont toutefois des spécialités si rares qu'on peut les négliger.

11. Comme dans la base Parchemin, les mentions professionnelles doubles ou triples sont nombreuses. Tel qui s'est dit taillandier le jour de ses noces est devenu armurier pour le baptême de son premier-né et meurt parfois... forgeron. Vu la confusion qui existe entre les appellations forgeron, taillandier et serrurier, il ne sert à rien de descendre au niveau spécifique déterminé par la lettre (o, n ou r) accompagnant le 2 . 
disons, entre un 20 (forgeron) et un $2 \mathrm{n}$ (taillandier) apparaissant concurremment sur une fiche.

Si le registre du PRDH est incomplet, contient-il par ailleurs des noms qui ne se retrouvent pas dans les actes notariés? Pour essayer de le savoir, nous avons extrait les listes des hommes auxquels un acte d'état-civil passé dans le gouvernement de Montréal avant 1766 avait attribué un des métiers du fer énumérés ci-dessus et avons comparé celles-ci à celles que nous avions constituées à partir des actes notariés.

Le taux de recoupement brut varie beaucoup selon le métier. La liste de mentions 2o (forgeron), par exemple, livre 117 noms différents; 100 ont été relevé aussi dans le registre des forgerons établi d'après la base Parchemin et deux autres, dans celui des taillandiers. Restent 15 sujets absents des actes notariés, mais mentionnés comme forgerons dans au moins un acte d'état-civil. Ce taux d'absence brut de $13 \%$ se réduit toutefois à $6 \%$ lorsqu'on examine les fiches individuelles. On constate alors que deux sujets ne sont jamais venus en Nouvelle-France (ce sont des pères de pionniers, et leur nom apparaît dans l'acte de mariage de leur enfant émigré), que trois résidaient en fait hors du gouvernement de Montréal, que deux apparaissent sous un nom différent dans les actes notariés, et qu'un était à l'époque un simple apprenti. Les sept derniers ne sont pas retraçables dans le registre du PRDH, soit parce qu'ils sont mal identifiés dans l'acte, soit parce qu'ils n'ont pas fait souche.

Pour les taillandiers, le PRDH recense 60 noms; 47 sont confirmés par les actes notariés. Des 13 absences, seulement trois (5\%) sont inexplicables.

En ce qui concerne les serruriers, 22 sujets sur 28 apparaissent dans nos listes, et un seul des six cas restants (4\%) ne peut être justifié.

Des 49 noms de la liste d'armuriers-arquebusiers (2l) fournie par le registre du PRDH, 44 figurent dans au moins un acte notarié, deux ne sont jamais venus au pays, et un apparaît sous un nom différent dans nos listes. Restent deux cas-mystères qui donnent un taux d'absence réel de $4 \%$.

Pour le groupe $2 \mathrm{~m}$ (ferblantiers-chaudronniers), la corrélation est parfaite. Les trois absences constatées parmi les 15 noms fournis par le PRDH proviennent d'une erreur de rédaction, d'une erreur de lecture et d'une mort si prématurée que l'artisan n'a sans doute jamais passé devant notaire. 


\section{L'EFFET DE LA PÉRIODE ET DE L'ORIGINE GÉOGRAPHIQUE}

La confusion observée entre les forgerons, les serruriers et les taillandiers est sans doute le reflet de la réalité économique: dans la région de Montréal, avant le milieu du XVIII ${ }^{e}$ siècle, les artisans du fer exerçaient tous les métiers à la fois, et seuls quelques armuriers trouvaient auprès de l'armée, et peut-être des équipeurs, de quoi alimenter une pratique plus spécialisée. Mais l'amalgame terminologique ne s'est peut-être pas fait tout de suite. Si tel est le cas,l'appellation forgeron devrait progresser avec le temps, soit parce qu'elle s'ajoute, soit parce qu'elle se substitue aux autres. Il ne faudrait pas y voir une déspécialisation des métiers, mais bien une adaptation du langage à la réalité coloniale.

Puisque le registre du PRDH fournit des indications fiables sur le métier, nous avons décidé de tester cette hypothèse à partir des codes 2o (forgeron). En gros, il s'agissait de savoir si la fréquence d'utilisation du titre avait crû ou décru avec le temps, si elle était plus grande chez les Canadiens ou chez les immigrants, et si elle avait eu tendance à remplacer les autres titres ou à s'y ajouter.

Nous avons restreint l'échantillon aux 98 forgerons titulaires d'au moins quatre actes notariés pour lesquels la base du PRDH avait livré au moins un code 2o: l'appellation était donc confirmée par deux sources complètement indépendantes. En ce qui concerne le nombre d'appellations professionnelles, nous avons établi trois catégories: code unique (2o), deux codes ( 20 et un autre), trois codes et plus ( 20 et deux ou trois autres). Pour mesurer l'effet de la période, nous avons séparé les sujets nés avant 1700 de ceux qui avaient vu le jour après cette date (et n'ont donc pu commencer leur carrière bien avant 1725). Enfin, nous avons distingué les immigrants européens des artisans nés au Canada.

\section{Tableau 6}

Répartition des codes 20 selon l'époque, l'origine (FR: France; NF: Nouvelle-France) et le nombre d'appellations

\begin{tabular}{lrrrrrrrrr}
\hline & \multicolumn{2}{c}{ Code unique } & \multicolumn{2}{c}{ Deux codes } & \multicolumn{2}{c}{ Trois codes et + } \\
& FR NF & Total (\%) & FR & NF & Total (\%) & FR & NF & Total (\%) \\
& 2 & 10 & $12(20)$ & 5 & 13 & $18(56)$ & 2 & 4 & $6(100)$ \\
Avant 1700 & 6 & 42 & $48(80)$ & 1 & 13 & $14(44)$ & 0 & 0 & $0(0)$ \\
Après 1700 & 8 & 52 & $60(100)$ & 6 & 26 & $32(100)$ & 2 & 4 & $6(100)$ \\
Total & & &
\end{tabular}


Des 98 sujets de l'échantillon, 42 (43\%) sont des Canadiens nés après 1700 qui emploient uniquement le titre de forgeron. Quand on sait à quel point les mentions uniques sont rares (parmi les 216 sujets de notre échantillon de validation, seulement un sur quatre s'en tient à un titre), ce degré de concentration paraît significatif. Chez les 36 artisans nés avant 1700 , le code 20 apparaît 12 fois seul (33\%), 18 fois en doublet $(50 \%)$ et six fois à l'intérieur d'une triple ou d'une quadruple mention (17\%). Or, l'échantillon compte 60 codes uniques $(61 \%), 32$ codes doubles (33\%) et six codes triples ou quadruples (6\%). Les premières générations d'artisans avaient donc tendance à jumeler le titre de forgeron à un ou à plusieurs autres alors que chez les forgerons qui ont commencé à travailler après 1720, les codes uniques représentent près de $80 \%$ du sous-échantillon, et on ne relève pas un triple ou quadruple code.

L'origine étrangère ne produit pas d'effet clair, mais ce souséchantillon est plutôt réduit: seulement 16 sujets sur 98 sont des immigrants. Ils se répartissent également entre code unique et codes multiples et presque également selon la période (neuf sont nés avant 1700 et sept après). Toutefois, les premières générations d'artisans canadiens étaient constituées d'une forte proportion de Français. La faible représentation numérique des immigrants dans le groupe des forgerons du XVII ${ }^{e}$ et du début du XVIII ${ }^{c}$ siècle trahit donc indirectement le peu d'attrait du titre pour les artisans d'origine européenne et confirme que forgeron est bien une étiquette fourre-tout qui s'est imposée au fil des décennies dans la région montréalaise ${ }^{12}$. Les premières générations, peut-être plus imprégnées de la mentalité corporatiste, sont restées attachées aux titres spécifiques. Dans leur cas, l'appellation forgeron est venue s'ajouter au titre initial, alors que pour les dernières générations du Régime français, la tendance est à la substitution pure et simple. D'où la proportion supérieure à la moyenne de codes multiples avant 1700 , et inférieure après.

\section{CONCLUSION}

Entreprise dans le but d'évaluer la fiabilité des mentions professionnelles du Registre de la population du Québec ancien - et dans l'espoir de pouvoir les utiliser pour dénombrer les effectifs des métiers du fer - notre analyse des actes notariés du gouvernement de Montréal nous mène à une conclusion un peu paradoxale. Le registre

12. Dans notre mémoire, nous avions déjà montré que l'appellation forgeron dissimulait une réalité très hétéroclite, à partir d'une analyse des outils de forge répertoriés dans les inventaires après-décès. Voir Dominique Bouchard, Le niveau de vie des artisans du fer à Montréal et à Québec entre 1730 et 1780 (Université de Montréal, 1992). 
du PRDH est fiable mais nettement incomplet. En revanche, le chercheur qui a la patience de dépouiller les rubriques de la base Parchemin peut obtenir une excellente estimation du nombre des artisans d'une catégorie donnée (bois, fer, cuir, etc.) établis dans la colonie ou dans un gouvernement ${ }^{13}$.

Les actes notariés ne semblent pas assez précis pour permettre de chiffrer les effectifs d'une ville (ils ne font pas toujours la distinction entre île et ville de Montréal, par exemple), mais un recoupement soigné avec les fiches du PRDH pourrait peut-être pallier cette faille. Ce qui est sûr, c'est que le métier (forgeron, menuisier, etc.) n'est pas un critère valable, car les conditions de vie dans la colonie ont déstructuré plus que la hiérarchie maître-compagnon-apprenti. En contraignant la plupart des artisans à la polyvalence, elles ont fait tomber les barrières traditionnelles entre les corporations d'un même secteur. Chez les artisans du fer, cela s'est traduit d'abord par un amalgame de titres, puis par l'apparition d'une appellation générique aux côtés de laquelle ont survécu les quelques dénominations des métiers spécialisés qui étaient réellement pratiqués.

À Montréal, cette évolution a produit la structure tripartite que nous avons décrite plus haut. Nous avons toutefois des raisons de croire que l'organisation des métiers du fer était différente à Québec: les serruriers y avaient probablement un statut distinct, à la manière des armuriers à Montréal ${ }^{14}$.

Il n'y a donc pas de solution «miracle» au problème de la structure et du dénombrement des artisans de la colonie. Chaque groupe est un cas d'espèce, chaque région a des spécificités socio-économiques suffisamment accentuées pour que les généralisations soient impossibles. Il faut procéder cas par cas. Notre étude a au moins démontré que la démarche est possible à partir des actes notariés.

13. Le critère que nous avons employé (le nombre d'actes) pour essayer de distinguer les artisans établis, qui ont exercé leur métier de façon continue pendant plusieurs années, sinon toute leur vie, dans le gouvernement de Montréal, donne un ordre de grandeur, pas un chiffre ferme. Pour le préciser, il faudrait reconstituer les carrières de tous nos sujets, ce qui est loin d'être fait. Nous croyons toutefois, d'après les données dont nous disposons sur l'échantillon traité dans cet article et sur les 325 sujets écartés, que la région de Montréal a dû compter de 200 à 250 artisans du fer sous le Régime français.

14. Les recherches que nous avons effectuées pour préparer notre mémoire nous ont permis de constater que l'appellation serrurier était beaucoup plus fréquente dans la capitale et l'outillage de ce métier, nettement mieux représenté dans les inventaires de Québec que dans ceux de Montréal. 